\title{
THEOREMS OF OSCILLATION FOR TWO LINEAR DIFFERENTIAL EQUATIONS OF THE SECOND ORDER WITH
}

\author{
TWO PARAMETERS*
}

BY

\author{
R. G. D. RICHARDSON
}

\section{Introduction.}

The present article deals primarily with the KLEIN oscillation theorem for the two differential equations with two parameters

$$
\begin{array}{ll}
\left(p_{1} u^{\prime}\right)^{\prime}+q_{1} u+\left(\lambda A_{11}+\mu A_{12}\right) u=0, & p_{1}(x)>0, \\
\left(p_{2} v^{\prime}\right)^{\prime}+q_{2} v+\left(\lambda A_{21}+\mu A_{22}\right) v=0, & p_{2}(y)>0,
\end{array}
$$

where the functions $p_{1}(x), q_{1}(x), A_{11}(x), A_{12}(x)$ and $p_{2}(y), q_{2}(y), A_{21}(y)$, $A_{22}(y)$ are supposed analytic in their respective intervals. What are the conditions for the existence of pairs of parameter values $\lambda, \mu$ for which exist solutions $u(x), v(y)$ which satisfy the boundary conditions

$$
u\left(a_{1}\right)=u\left(b_{1}\right)=0, \quad v\left(a_{2}\right)=v\left(b_{2}\right)=0,
$$

and oscillate $m$ and $n$ times respectively in the intervals $a_{1} b_{1}$ and $a_{2} b_{2}$ ? Such a problem arises naturally in the study of mathematical physics. $\dagger$ The intimate connection between this problem and existence theorems for automorphic functions has been discussed by KLEIN $\ddagger$ and HiLb.§

The first problem of this type was proposed by KLeIN in connection with the solutions of the Lamé equation; this and similar problems of a more general type have been treated by BôcHER. \|

* Presented to the Society December 28, 1910, and April 28, 1911.

$\dagger$ For references see the article contributed by BôchER to the Encyklopïdie der Mathematischen Wissenschaften (II A 7a).

$\ddagger$ Bemerkungen zur Theorie der linearen Differentialgleichungen zweiler Ordnung, M a t h ematische Annalen, vol. 64 (1907), p. 175.

$\$ \ddot{U b e r}$ Kleinsche Theoreme in der Theorie der linearen Differentialgleichungen, $\mathrm{M}$ a thematische Annalen, vol. 66 (1908), p. $215 . \quad$ Zweite Mitteilung, vol. 68 (1910), p. 24.

$\|$ Bulletin of the American Mathematical Society, ser. 2, vol. 4 (1898), p. 295 and p. 365 , vol. 5, p. 22. A very full list of references will be found in the article in the Encyklopädie, loc. cit. 
Hilbert * has recently treated the problem of the existence of solutions of equations $(A)$ for the case $q_{1}=q_{2}=0, A_{12}>0, A_{22}<0$. By means of the transformation $z(x, y)=u(x) v(y)$ the two equations $(A)$ may be transformed into the partial differential equation

$$
-A_{22}\left(p_{1} z_{x}\right)_{x}+A_{12}\left(p_{2} z_{y}\right)_{y}+\lambda\left(A_{21} A_{12}-A_{11} A_{22}\right) z=0 \text {. }
$$

Since this equation is of the elliptic type, it follows that the parameter $\lambda$ can be determined in an infinite number of ways such that the corresponding solutions vanish on the boundary of the rectangle. Hilbert shows that for these same values of $\lambda$ and the corresponding values of $\mu$ there are solutions of the equations $(A)$; but no oscillation theorems are derived. Since the present article was written, Jitsuo Yoshikawa, $\dagger$ under the direction of Hilbert, has proved the oscillation theorem for the special case $q_{1}=q_{2}=0, A_{12}>0, A_{22}<0, A_{11}>0$, $A_{21}>0$.

In $\$ 4$ of this article necessary conditions and sufficient conditions have been derived for the existence of solutions of the general equations $(A)$, subject to the given boundary conditions. Sufficient conditions for the uniqueness of solutions are also derived. The discussion of the oscillation theorem for the equation with one parameter $\left(p y^{\prime}\right)^{\prime}+q y+\lambda k y=0$ is extended in $\S 3$ to the case not hitherto treated, viz., when $q(x)$ is positive in at least a part of the interval and $k(x)$ takes on both signs.

Although the treatment here given is entirely independent of such methods, the results were first obtained by means of the Calculus of Variations. This is another illustration of the fact emphasized by HILBERT that the calculus of variations has an intimate relation to differential equations and to integral equations and is more fundamental as a discipline than either of these theories. The relation between the oscillation theory for one equation with one parameter and the calculus of variations problem was discussed by the author in the first of a series of articles $\ddagger$ in the Mathematische Annalen; a second article which treats of the corresponding problem for two equations with two parameters is to appear shortly.

It is interesting to note that when the equations $(A)$ (for $q_{1}=q_{2}=0$ ) have solutions of the type sought, the partial differential equation $(B)$ has an infinite number of solutions which vanish all around the rectangle. Since, as we shall prove, $A_{22}, A_{12}$ may be arbitrary, except that not both are identically zero, we

* Grundzüge einer allgemeinen Theorie der linearen Integralgleichungen, 6. Mitteilung, Göttinger Nachrichten (1910).

$\dagger$ Ein zweiparametriges Oszillationstheorem, Göttinger Nachrichten (1910) (appeared February, 1911).

$\ddagger$ Das Jacobische Kriterium der Variationsrechnung und die Oszillationseigenschaften linearer Differentialgleichungen 2. Ordnung, Mathematische Annalen, vol. 68 (1910), p. 279. 
have an example of a partial differential equation of a general type for which a double infinity of solutions exist which vanish all around the boundary.

Several problems naturally suggest themselves in connection with this discussion. Among these is the expansion of functions of two variables in terms of the products of the solutions of the equations $(A)$ and the extension of the theory of oscillation to the following cases: (1) when the boundary conditions are modified; (2) when one or more of the functions $q_{1}, q_{2}, A_{11}, A_{12}, A_{21}, A_{22}$ becomes infinite; (3) when there are three equations and three parameters; (4) when there is one partial differential equation and one parameter. The discussion of (3) will appear in the Mathematische Annalen; I hope to take up the other problems at a later date.

\section{$\S 1$. The equation of the second order with one parameter.}

Let $p(x)>0, q(x)$, and $k(x)$ be analytic functions of $x$ in the interval 0 1. Do there exist parameter values $\lambda$ for which the linear self-adjoint differential equation

$$
\left(p y^{\prime}\right)^{\prime}+q y+\lambda k y=0
$$

has solutions $y(x)$ which satisfy the boundary conditions *

and oscillate $n$ times?

$$
y(0)=y(1)=0
$$

In the discussion of this article certain results which have been already established in regard to this oscillation problem are necessary. These are as follows:

$(A) \dagger$. If $k(x) \geqq 0$, there exist an infinite number of characteristic values (Eigenwerte) $\lambda=\lambda_{1}, \lambda_{2}, \cdots\left(\lambda_{1}<\lambda_{2}<\lambda_{3} \cdots\right)$, for each of which there is a solution of the differential equation (1) subject to the boundary conditions (2). These characteristic functions (Eigenfunktionen) $y=Y_{i}(x)$ oscillate $i-1$ times in the interval 01 . A continuous increase of $\lambda k(x)$ moves continuously to the left all the succeeding zeros of that solution of (1) which vanishes at $x=0$. Of the characteristic numbers some may be negative but the aggregate has a limiting point only at plus infinity.

$(B) \ddagger$. If $q(x) \leqq 0$ and $k(x)$ takes on both signs in the interval, there are

* Among the articles discussing this problem for more general boundary conditions may be mentioned those by Mason (these Transactions, vol. 7 (1906), p. 337) and BirkHoFF (these Transactions, vol. 10 (1909), p. 259).

(Added December 6.) In this connetion it may be well to note that HAOPT in his article Untersuchungen über Oszillationstheoreme (Würzburg dissertation, Teubner, 1911) has overlooked these papers. The main theorem of the first part of the dissertation (p. 35) is a special case of BirkHOFF's oscillation theorem (loc. cit., p. 269).

†Sturm, Journal de Mathématiques, vol. 1 (1836), p. 106. This and more general theorems of the same type have been established on a rigorous basis by BôcHER, loc. cit.

$\ddagger$ HILBERT, Grundzüge einer allgemeinen Theorie der linearen Integralgleichungen, G öt tinger Nachrichten. 1. und 2. Mitteilung, 1904; 4. und 5. Mitteilung, 1906. 
an infinite number of positive and an infinite number of negative characteristic values $\lambda=\lambda_{1}, \lambda_{2}, \cdots ; \lambda_{1}, \lambda_{-2}, \cdots$ for each of which there exists a solution $Y_{i}(x)$ or $Y_{-i}(x)$ of the equation (1) subject to the condition (2). In both cases the aggregate of characteristic values have a limiting point only at infinity, and the solutions oscillate $i-1$ times in the interval.

$(C)^{*}$. When $q(x) \leqq 0$ and $k(x)$ is arbitrary, a continuous increase of $\lambda(\lambda>0)$ or a continuous decrease of $\lambda(\lambda<0)$ moves continuously to the left all the succeeding zeros of that solution of (1) which vanishes at $x=0$.

$(D)$. If the function $k(x)$ is made to vary continuously in the interval 01 , the parameter values $\lambda$, for which solutions vanishing at $x=0$ and $x=1$ exist, vary continuously. When $\lambda$ varies continuously, any solution of equation (1) for which $y(0)=0, y^{\prime}(0)=$ const. varies continuously.

To extend the above results we prove the following theorem:

(E) When $q(x)$ is positive in at least a part of the interval and $k(x)$ takes on both signs, the zero at $x=1$ of that solution $y(x)$ of the equation (1) which vanishes at $x=0$ may be moved to the right or left by a change of the parameter $\lambda$ unless

$$
\int_{0}^{1} k y^{2} d x=0 .
$$

For, $\uparrow$ if we denote by $y$ and $y^{*}$ those solutions of (1) corresponding to $\lambda$ and $\lambda+\epsilon$ respectively for which $y^{\prime}(0)=y^{* \prime}(0)=1$ and multiply the equations

$$
\begin{aligned}
\left(p y^{\prime}\right)^{\prime}+q y+\lambda k y & =0 \\
\left(p y^{* \prime}\right)^{\prime}+q y^{*}+(\lambda+\epsilon) k y^{*} & =0
\end{aligned}
$$

by $y^{*}$ and $y$ respectively, subtract and integrate from 0 to 1 , we obtain the relation

$$
p(1) y^{\prime}(1) y^{*}(1)=\epsilon \int_{0}^{1} k y y^{*} d x
$$

* Richardson, loc. cit.

† This proof is a modification of that given by the author for a slightly different case, loc. cit., Satz 3. The reasoning applies equally well to equation (1) for the cases discussed in $(A)$ and $(C)$. In $(A)$, however, since $k$ is positive,

$$
\int_{0}^{1} k y^{2} d x
$$

cannot be zero. To show that this is also true in case $(C)$, it is only necessary to multiply the equation by $y$, to integrate from 0 to 1 , and in the resulting relations

$$
\int_{0}^{1}\left(p y^{\prime 2}-q y^{2}\right) d x=\lambda \int_{0}^{1} k y^{2} d x
$$

to note that since $p>0, q \leqq 0$, the first integral is positive. 
From (I)) it follows that this may be written

$$
p(1) y^{\prime}(1) y^{*}(1)=\epsilon\left[\int_{0}^{1} k y^{2} d x+\epsilon^{\prime}\right],
$$

where $\epsilon^{\prime}$ is an infinitesimal of the same order as $\epsilon$. In case the integral is different from zero, by giving $\epsilon$ the proper sign, $y^{\prime}(1) y^{*}(1)$ can be made positive. If $x=1$ is the first, third, . . zero of $y(x)$, it follows that $y^{\prime}(1)$ is negative and hence $y^{*}(1)$ is negative and the zero of $y^{*}$ is to the left of $x=1$; if $x=1$ is the second, fourth, . . . zero of $y(x), y^{\prime}(1)$ is positive and hence $y^{*}(1)$ is positive and the zero of $y^{*}$ is again to the left. By choosing $\epsilon$ of contrary sign the zero may be moved to the right.

\section{$\S 2$. The equation of the second order with two parameters.}

If in the equation $\left(p y^{\prime}\right)^{\prime}+q y+\lambda k y=0$ of the preceding section we set $k(x)=A_{1}(x)+\kappa A_{2}(x)$ where $A_{1}(x)$ and $A_{2}(x)$ are analytic functions not identically zero and $\kappa$ an arbitrary parameter, it is evident that, at least when $q(x) \leqq 0$, the parameter $\lambda$ is a function of $\kappa$ which is one or two-valued according as $A_{1}+\kappa A_{2}$ takes on one or both signs. On setting $\mu=\kappa \lambda$, it follows that the linear differential equation with two parameters

(3) $\left(p y^{\prime}\right)^{\prime}+q y+\left(\lambda A_{1}+\mu_{1} 1_{2}\right) y \equiv\left(p y^{\prime}\right)^{\prime}+q y+\lambda\left(A_{1}+\kappa A_{2}\right) y=0$,

has for any fixed ratio between the parameters $\lambda$ and $\mu$ one or two solutions which satisfy the boundary conditions $y(0)=y(1)=0$ and do not vanish within the interval 01 , one or two solutions which vanish once within the interval, and, in general, one or two solutions which vanish $n$ times within the interval. If a solution is demanded which vanishes at $x=0, x=1$, it is evident that a change of one parameter involves a change of the other. Since $\lambda$ is a continuous function of $\kappa(\S 1, J)$, it is evident that for any fixed value of $n, \lambda$ and $\mu$ may be regarded as continuous * functions of one another except when one or both become infinite. We proceed now to a more complete determination of the nature of the function $\mu(\lambda)$, assuming throughout this section that $q(x) \leqq 0$. The derivative $d \mu / d \lambda$ is an analytic function except when it becomes infinite. For on differentiating the equation (3) with regard to $\lambda$ we have

$$
\left(\begin{array}{c}
\partial y^{\prime} \\
\partial \lambda
\end{array}\right)+q \frac{\partial y}{\partial \lambda}+\left(\lambda A_{1}+\mu A_{2}\right) \frac{\partial y}{\partial \lambda}+A_{1} y+A_{2} y \frac{d \mu}{d \lambda}=0
$$

On multiplying (3) by $\partial y / \partial \lambda$ and (4) by $y$, subtracting the results, and integrating from 0 to 1 we have

* To prove that this is true for $\kappa= \pm \infty$, it is only necessary to set $\lambda A_{1}+\mu A_{2}=\mu\left(A_{2}+h A_{1}\right)$ and to note that $\mu(h)$ is continuous for $h=0$. Hence also $\lambda(\kappa)$ is continuous and

$$
\lambda(+\infty)=\lambda(-\infty) \text {. }
$$




$$
\frac{d \mu}{d \lambda}=-\frac{\int_{0}^{1} A_{1} y^{2} d x}{\int_{0}^{1} A_{2} y^{2} d x}, \quad \frac{d \lambda}{d \mu}=-\frac{\int_{0}^{1} A_{2} y^{2} d x}{\int_{0}^{1} A_{1} y^{2} d x} .
$$

Since $\mu=\lambda_{\kappa}$, it may be shown that

$$
\frac{d \lambda}{d \kappa}=-\frac{\lambda \int_{0}^{1} A_{2} y^{2} d x}{\int_{0}^{1}\left(A_{1}+\kappa A_{2}\right) y^{2} d x}, \quad \frac{d \mu}{d \kappa}=\frac{\lambda \int_{0}^{1} A_{1} y^{2} d x}{\int_{0}^{1}\left(A_{1}+\kappa A_{2}\right) y^{2} d x} .
$$

Since $y$ is a continuous function of $\lambda(\S 1, D)$ and since both of the integrals in (5) cannot be zero at the same time, it follows that the curve $\mu(\lambda)$ has a continuously turning tangent.

If $A_{1}(x) / A_{2}(x)=c$ (constant), it follows from (j) that $d \mu / d \lambda=-c$ and that $\mu(\lambda)$ is a straight line. As $n$ increases, the absolute value of the parameter $c \lambda+\mu$ increases $(\S 1, A)$; and the minimum distance of the line from the origin increases without limit. If $A_{2}$ has only one sign, there is only one set of lines $\mu(\lambda)$ which cuts the $\mu$ axis above or below the origin according as this sign is positive or negative. If $A_{2}$ has both signs, both sets of lines occur.

Proceeding now to the discussion of the cases where $A_{1}(x) / A_{2}(x)$ is not a constant, let us investigate the nature of $A_{1}+\kappa A_{2}$ regarded as a function of $\kappa$. We shall first consider that $A_{2}(x) \geqq 0$; by an interchange of the parameters or by a change of sign of one of them, all cases where one of the functions $A_{1}, A_{2}$ has one sign may be reduced to this. There is an interval ${ }^{*} \kappa_{1} \kappa_{2}$ of values of $\kappa$ for which $A_{1}+\kappa A_{2}$ takes on both signs and for which there are two sets of characteristic numbers ( $\lambda$ and $\lambda_{-}$); that is, in the $\lambda \mu$ plane a straight line through the origin with a slope $\kappa\left(\kappa_{1}<\kappa<\kappa_{2}\right)$ cuts any curve $\mu(\lambda)$ in two points. For any value of $\kappa$ in the interval $\kappa_{2} \infty \kappa_{1}$ such a line will cut any curve $\mu(\lambda)$ but once.

It is necessary to determine more exactly the form of the curve $\mu(\lambda)$. On multiplying equation (3) by $y$ and integrating we obtain the relation

$$
\int_{0}^{1}\left(p y^{\prime 2}-q y^{2}\right) d x=\lambda \int_{0}^{1}\left(A_{1}+\kappa A_{2}\right) y^{2} d x .
$$

Since $p>0, q \leqq 0$, it follows that the integral on the right has the same sign as $\lambda$ and cannot be zero unless $y \equiv 0$. Hence from (6) $d \lambda / d \kappa$ is always negative. The positive characteristic value $\lambda$ is a single-valued decreasing function of $\kappa$ in the interval, $\kappa_{1} \infty$ and if it becomes infinite, it must do so for the value $\kappa=\kappa_{1}$. To show that it actually becomes infinite it is only necessary to note

${ }^{*}$ It is readily seen that $\kappa_{1}=-\max \left[A_{1}(x) / A_{2}(x)\right], \kappa_{2}=-\min \left[A_{1}(\cdot) / A_{2}(x)\right]$. 
that for values of $\kappa$ in the interval $\kappa_{1} \kappa_{1}+\epsilon$ the function $A_{1}+\kappa A_{2}$ in (7) is positive only in a small portion of the interval 01 , and that the integral of this function, being positive, must be an infinitesimal. The integral on the left of (7) being a finite quantity, $\lambda$ must increase without limit. In a similar manner $\lambda_{L}$ is a single-valued decreasing function of $\kappa$ in the interval $-\infty \kappa_{2}$ and becomes infinite for $\kappa=\kappa_{2}$. When $\lambda=0$, the values of $\mu$ for which equation (3) has a solution are positive $(\S 1, A)$; and since $\kappa_{1} \leqq-A_{1} / A_{2} \leqq \kappa_{2}$ it follows from (5) that $\kappa_{1} \leqq d_{\mu} / d \lambda \leqq \kappa_{2}$. Hence the curves $\mu(\lambda)$ cut the $\lambda$ axis above the origin and recede always from the half-lines $\mu=\kappa_{1} \lambda, \mu=\kappa_{2} \lambda$ though approaching parallelism to them. For any fixed values of $\kappa$ an increase of the number $n$ of oscillations gives $(\S 1, A, B)$ a corresponding increase to $\lambda$ and to $-\lambda$. Hence the curves $\mu(\lambda)$ corresponding to the various values of $n$ never cut one another and as $n$ increases the minimum distance from the origin increases without limit.

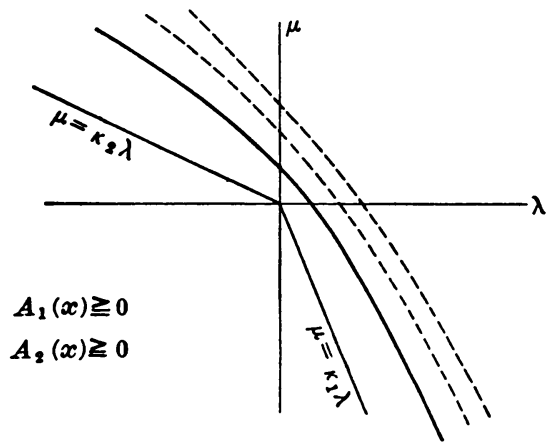

FIG. 1.

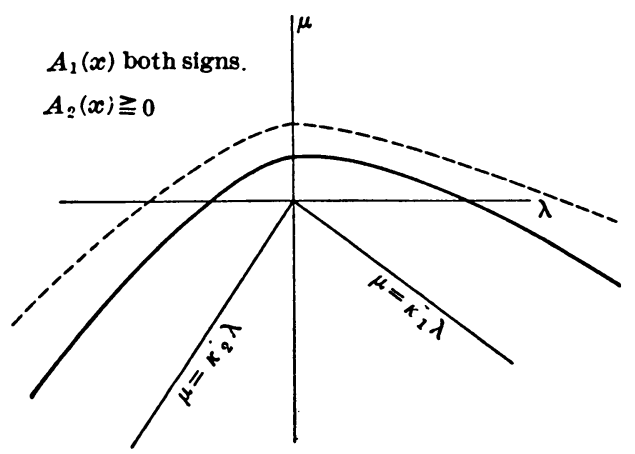

Fig. 2.

When $A_{1} \geqq 0$, it follows from (5) that $\mu(\lambda)$ is a continuously decreasing function (Fig. 1). The dotted lines indicate the curves $\mu(\lambda)$ for greater values of $n$. If $A_{1}>0$ and $A_{2} \geqq 0$ but equal zero for some value of $x$ in the interval 01 , the value of $\kappa_{1}$ is $-\infty$. In this case the curve $\mu(\lambda)$ approaches parallelism to the $\mu$ axis. Since, however, for a value of $\lambda$ great at pleasure there is a corresponding value of $\mu$, the curve recedes indefinitely from this axis. When $A_{2}>0$ and $A_{1} \geqq 0$ but equals zero for some value of $x$, the value of $\kappa_{2}$ is zero and the curve $\mu(\lambda)$ although receding indefinitely from the axis of $\lambda$ approaches paralellism to it. If $A_{1} \geqq 0, A_{2} \geqq 0$ but one of the functions becomes zero at one point and the other at another point of the interval 01 , then $\kappa_{1}=-\infty$ and $k_{2}=0$; the curve recedes indefinitely to the right of the $\mu$ axis and above the $\lambda$ axis but approaches parallelism to these lines.

Fig. 2 shows the form of the curves $\mu(\lambda)$ for the case when $A_{1}$ takes on both signs. $\mu$ is a single-valued function of $\lambda$ and attains its maximum for some function $y(x)$ which makes 


$$
\int_{0}^{1} A_{1} y^{2} d x=0
$$

(Formula 5). When this integral is positive, the function $\mu(\lambda)$ decreases and when it is negative, $\mu(\lambda)$ increases.

There still remains to be considered the case where $A_{1}$ and $A_{2}$ both take on both signs in the interval. There are here two possibilities; either there exists an interval $\kappa_{2} \kappa_{1}$ (which evidently contains neither $\kappa=0$ nor $\kappa=\infty$ ) for which $A_{1}+\kappa A_{2}$ takes on one sign only, or $A_{1}+\kappa A_{2}$ takes on both signs for all values of the parameter $\kappa$.

In the former case, for some values of $\lambda, \mu(\lambda)$ is a two valued function ( $\$ 1$, $B$ ) having a maximum or minimum when the solution is such a function $y(x)$ that

$$
\int_{0}^{1} A_{1} y^{2} d x=0
$$

(Formula 5 ), and for some value of $\mu, \lambda(\mu)$ is a two-valued function having a maximum or minimum when

$$
\int_{0}^{1} A_{2} y^{2} d x=0 .
$$

Since Formula 5 can be established for this case, the curve $\mu(\lambda)$ is of the same general form as in the other cases, the slope never taking on any value intermediate between $\kappa_{2}$ and $\kappa_{1}$. The reasoning which in the other cases showed that $\lambda$ is a monotone function of $\alpha$ is here no longer valid; but since $d \lambda / d_{\kappa}$ has only one sign within the interval $\kappa_{2} \kappa_{1}$ (Formula 6), $\lambda^{\cdot}$ a monotone

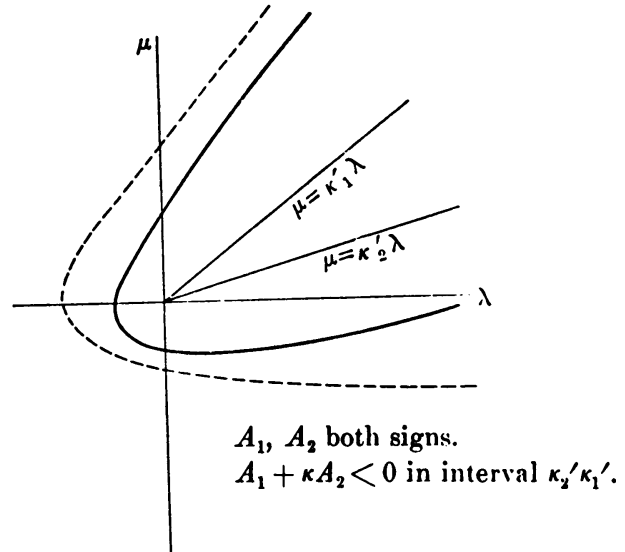

Fig. $3 a$.

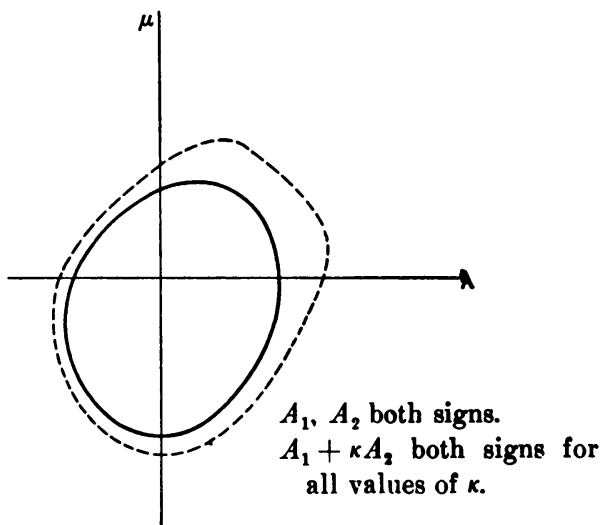

Fig. $3 b$.

function of $\alpha$ in this interval. Fig. $3(a)$ is a graph of the function $\mu(\lambda)$ for the case that in the interval $\kappa_{2}^{\prime} \kappa_{1}^{\prime}\left(\kappa_{1}^{\prime}>\kappa_{2}{ }^{\prime}\right)$ of positive values of $\kappa$, the function $A_{1}+\kappa A_{2}$ is negative for all values of $x$. If for a positive interval of $\kappa$ the func- 
tion $A+\kappa A_{2}$ is positive, the half-lines lie in the third quadrant; if the $\kappa$ interval is negative, the half-lines lie in the second or fourth quadrant according as $A_{1}+{ }_{\kappa} A_{2}$ is negative or positive throughout the interval of $x$.

If for all values of $\kappa$ the function $A_{1}+\kappa A_{2}$ takes on both signs in the interval, there exists $(\S 1, B)$ for any number $n$ of oscillations of the solution of (3) two characteristic numbers $\left(\lambda\right.$ and $\left.\lambda_{-}\right)$. The value of $\lambda$ is always finite and in an analogous manner it is seen that $\mu$ is always finite. The curve $\mu(\lambda)$ is then continuous (Fig. $3(b)$ ) and of such a nature that it is cut twice and only twice by any straight line passing through the origin. For any fixed value of $\kappa$ an increase of the number $n$ of oscillations gives $(\S 1, B)$ a corresponding increase to $\lambda$ (or to $-\lambda_{-}$). The curves $\lambda(\mu)($ or $\mu(\lambda))$ corresponding to the various values of $n$ never cut one another, and for sufficiently large $n$ the minimum distance from the origin to the curve corresponding to $n$ is large at pleasure.

\section{\$3. The oscillation theorem for the general self-adjoint equation of the second order with one or two parameters.}

Given the most general self-adjoint differential equation of the second order containing one parameter

$$
\left(p y^{\prime}\right)^{\prime}+q y+\lambda k y=0, \quad p(x)>0,
$$

do there always exist one or more values of the parameter $\lambda$ such that the corresponding solution satisfies the boundary conditions $y(0)=y(1)=0$ and oscillates $n$ times in the interval ? Since such a theorem has already been established $(\S 1, A)$ for the case that $k(x)$ has one sign and $q(x)$ is arbitrary and $(\S 1, B)$ for the case $q(x) \leqq 0$ and $k(x)$ arbitrary, it is necessary to investigate only the case where $k(x)$ takes on both signs in the interval and $q(x)$ is positive in at least a portion of the interval.

That for all values of $n$ sufficiently large this oscillation theorem is valid may be seen as follows. Let us denote by $n_{1} \geqq 0$ the number of zeros within the interval 01 of that solution of the equation $\left(p y^{\prime}\right)^{\prime}+q y=0$, which vanishes at $x=0$. If the $\left(n_{1}+1\right)$ th zero is at $x=1$, there is for $\lambda=0$ a solution of (8) which oscillates $n_{1}$ times in the interval. If the $\left(n_{1}+1\right)$ th zero is outside the interval 01 , the equation

$$
\left(p y^{\prime}\right)^{\prime}+\mu q y+\lambda k y=0
$$

has for $\lambda=0, \mu=\bar{\mu}>1(\S 1, D)$ a solution which oscillates $n$ times and satisfies the boundary conditions. For $\mu=0$ there are two values of $\lambda$ which give such a solution ( $\S 1, \mathrm{~B})$, and since $\mu$ is a continuous function of $\lambda(\S 2)$ there will be at least two values of $\lambda$ for which $\mu=1$. See Fig. 2, 3(a), 3(b). For these values of $\mu$ and $\lambda$ the equations (8) and (9) are identical. The same method of reasoning shows that for all values of $n$ greater than $n_{1}$ there are at least two 
values of $\lambda$ for which solutions of the type required exist. On the other hand there are cases where, for small values of $n$, no solution exists which satisfies. the boundary conditions. If, for example, $q \geqq 0$ it may be seen from Fig. 2 that for $\mu$ large enough there is no characteristic number $\lambda$ which satisfies equation (9). On setting $\mu q=\bar{q}$ there results an equation

$$
\left(p y^{\prime}\right)^{\prime}+\bar{q} y+\lambda k y=0,
$$

which has no solution for $n=0$. There will be a smallest number $n=\bar{n}$ for which equation (9), for $\mu=1$ (that is, equation 8 ), has a solution of the type sought; for this and larger values of $n$ there will be at least two characteristic numbers. By increasing or decreasing $\lambda$ the $\left(n_{1}+1\right)$ th zero $x_{n+1}$ of that solution of (9) which vanishes at $x=0$ can be so determined that $x_{n+1} \geqq 1$ while the $n$th zero $x_{n}$ attains its greatest value $(\S 1, E)$ when

$$
\int_{0}^{x_{n}} k y^{2} d x=0 \text {. }
$$

We proceed now to the general equation with two parameters

$$
\left(p y^{\prime}\right)^{\prime}+q y+\left(\lambda A_{1}+\mu A_{2}\right) y \equiv\left(p y^{\prime}\right)^{\prime}+q y+\lambda\left(A_{1}+\kappa A_{2}\right) y=0 \text {. }
$$

Where $q(x)$ is positive in at least a part of the interval, it follows from the preceding discussion that if $A_{1}+\kappa A_{2}$ takes on both signs for all values of $\kappa$, then in some cases it is not possible to obtain a parameter value $\lambda$ for which there are solutions which oscillate a small number of times. With an increase of $q(x)$ the inside oval in Fig. 3(b) has shrunk up to a single point and disappeared. In all other cases, that is when for somè values of $\kappa$ the function $A_{1}+\kappa A_{2}$ has one sign, there exist sets of values $\lambda, \mu$ such that the corresponding solutions of (10) oscillate $n$ times $(n=0,1,2 \cdots)$. The derivation of Formulæ 5 is entirely independent of the sign of $q(x)$ and the asymptotic directions cannot lie without the interval $\kappa_{1} \kappa_{2}$. Let us compare them with the asymptotic directions of the curve $\bar{\mu}(\lambda)$ for the related equation

$$
\left(p y^{\prime}\right)^{\prime}+\bar{q} y+\left(\lambda A_{1}+\mu A_{2}\right) y=0, \quad \bar{q}(x)>0, \quad \bar{q}(x)>q(x) .
$$

These latter lie entirely outside the half-lines $\mu=\lambda \kappa_{1}, \mu=\lambda \kappa_{2}$ and become parallel to them at infinity $(\S 2)$. Since $\bar{q}(x)>q(x)$ the zeros of the corresponding solutions of $(10)$ and (11) cannot coincide $(\S 1, A)$ and the curves $\mu(\lambda)$ and $\bar{\mu}(\lambda)$ cannot meet. Since the asymptotic directions of $\mu(\lambda)$ cannot then lie outside these half lines nor inside them, they must coincide with them. These curves $\mu(\lambda)$ which we have proved to exist for all values of $n$ (except in the special case where $A_{1}+\kappa A_{2}$ has both signs for all values of $\kappa$ ) have the same asymptotic direction (which is also independent of $q(x)$ ). For $n<n_{1}$ it is easily seen that these curves lie in part within the half-lines. For, if e. $g$. 
$A_{2} \geqq 0$ (Fig. 2), equation 10 has for $\lambda=0$ and $n<n_{1}$ a solution when $\mu<0$ $(\S 1, A)$.

\section{§4. The oscillation theorem for two equations of the second order with two parameters.}

We proceed now to the discussion * of the oscillation theorem for the two linear self-adjoint differential equations

$$
\begin{aligned}
\left(p_{1} u^{\prime}\right)^{\prime}+q_{1} u+\left(\lambda A_{11}+\mu A_{12}\right) u & =0, \\
\left(p_{2} v^{\prime}\right)^{\prime}+q_{2} v+\left(\lambda A_{21}+\mu A_{22}\right) v & =0,
\end{aligned}
$$

where it is assumed that $p_{1}(x)>0, q_{1}(x), A_{11}(x), \dot{A_{12}}(x), p_{2}(y)>0$, $q_{2}(y), A_{21}(y), A_{22}(y)$ are analytic functions, and that neither both the functions $A_{11}, A_{12}$ nor both the functions $A_{21}, A_{22}$ are identically zero. It is now possible to determine necessary conditions and sufficient conditions for the existence of parameter values $\lambda, \mu$ for which exist solutions $u(x), v(y)$ that satisfy the boundary conditions

$$
u\left(a_{1}\right)=u\left(b_{1}\right)=0, \quad v\left(a_{1}\right)=v\left(b_{1}\right)=0,
$$

and which oscillate $m$ and $n$ times respectively $\left(m \geqq m_{1}, n \geqq n_{1}\right)$ in the intervals.

In considering the graphs ( $\S \S 2-3$ ) of the curves $\mu(\lambda), \bar{\mu}(\lambda)$ corresponding respectively to a solution of the first equation $(A)$ oscillating $m$ times and to one of the second equation oscillating $n$ times it is clear that when one of the curves is an oval (Fig. $3(b)$ ), there is a double infinity of values $m, n$ for which there is no solution. For, if the curve $\mu(\lambda)$ is an oval, i. e., if, for all values of $\kappa$, the function $A_{11}+\kappa A_{12}$ takes on both signs, then for $m$ fixed it has been shown in $\S 2$ that the value of $n$ can be taken so large that the graph of the function $\mu(\lambda)$ is everywhere at a distance from $(0,0)$ greater than that of the most remote point of $\mu(\lambda)$. Hence in order that there be solutions of $(A)$ of the type required it is necessary that for at least one value of $\kappa$ the function $A_{11}+\kappa_{*} \mathrm{~A}_{12}$ and for at least one value of $\kappa$ the function $A_{21}+\kappa A_{22}$ have each only one sign for all values of $x$ in their respective intervals.

It is then clear that if solutions of $(A)$ of the type sought are to exist there must be a pair of half-lines corresponding to each of the curves $\mu(\lambda), \bar{\mu}(\lambda)$ (see Figs. 1, 2, 3a). There are three possibilities: of these sets of half-lines one may (1) cut, (2) lie outside, (3) lie inside the other. As limiting cases, one or both the arms may coincide with one of the other set.

In case (3) it can be shown that there are an infinite number of curves of one set which do not cut any of those of the other and that the oscillation theorem is not valid.

* The particular method of attack in this section, which is much more concise and elegant than that originally developed by the author, was suggested by Professor Birkнoff. 
For let us denote by $m_{1}$ the number of zeros within the interval $a_{1} b_{1}$ of that solution of $\left(p_{1} u^{\prime}\right)^{\prime}+q_{1} u=0$ which vanishes at $x=a_{1}$ and by $n_{1}$ the number of zeros within the interval $a_{2} b_{2}$ of that solution of $\left(p_{2} v^{\prime}\right)^{\prime}+q_{2} v=0$ which vanishes at $y=a_{2}$ and, in order to fix the ideas, assume that the arms correponding to $\mu(\lambda)$ are within the arms corresponding to $\bar{\mu}(\lambda)$. Then the curve $\bar{\mu}(\lambda)$ lies outside the curve $\mu(\lambda)$ for $m=m_{1}$, except perhaps in a limited region about the origin. For sufficiently large $n$ the corresponding curve $\bar{\mu}(\lambda)$ lies at an arbitrarily great distance from the origin and hence entirely outside the curve $\mu(\lambda)$ for $m=m_{1}(\S 2)$ :

In case (1) there is evidently at least one solution for all values of $m$ and $n$. Since there is at least one value of $\kappa$ for which $A_{11}+\kappa A_{12}$ and $A_{21}+\kappa A_{22}$ have one and the same sign, by a linear transformation of the parameters $\lambda$ and $\mu$, $A_{12}$ and $A_{22}$ can be made to have one and the same sign. If now

$$
\frac{A_{11}(x)}{A_{12}(x)} \neq \begin{aligned}
& A_{21}(y) \\
& A_{22}(y)
\end{aligned}
$$

for all values of $x$ and $y$, it is evident that the relation

$$
\frac{\int_{a_{1}}^{b_{1}} A_{11} u^{2} d x}{\int_{a_{1}}^{b_{1}} A_{12} u^{2} d x}=\frac{\int_{a_{2}}^{b_{2}} A_{21} v^{2} d y}{\int_{a_{2}}^{b_{2}} A_{22} v^{2} d y}
$$

is impossible for any functions $u(x), v(y)$, and that the curves $\mu(\lambda), \bar{\mu}(\lambda)$ having throughout their lengths slopes entirely different from each other (Formula 5) can cut in one point only. Hence in case (1) a sufficient condition for the uniqueness of solutions of equation $(A)$ for fixed values $m, n$ is that*

$$
A_{12}(x) A_{21}(y)-A_{11}(x) A_{22}(y) \neq 0 \text {. }
$$

That this may be written

$$
\min \left(A_{11} / A_{12}\right)>\max \left(A_{21} / A_{22}\right) \text { or } \min \left(A_{21} / A_{22}\right)>\max \left(A_{11} / A_{12}\right)
$$

is readily seen. The result may also be stated as follows: It is sufficient that there exist a straight line through the origin each half of which lies within one set of half-lines; in other words that there exist a value of $\kappa$ such that $A_{11}+\kappa A_{12}$ and $A_{21}+{ }_{\kappa} A_{22}$ each have one sign, but these opposite.

Since in case (2) for $m \geqq m_{1}, n \geqq n_{1}$ the curves $\mu(\lambda), \bar{\mu}(\lambda)$ lie outside the half-lines, it is clear that there are at least two solutions of the type sought. If a straight line can be drawn through the origin each half of which lies within one set of half-lines, in other words if there exists a value of $\kappa$ such that $A_{11}+\kappa A_{12}$

* This relation is invariant under the parameter transformation and may be applied to the original equation. 
and $A_{21}+\kappa A_{22}$ each have one sign, but those opposite, then, by a linear transformation of parameters, it is possible to make $A_{12} \geqq 0, A_{22} \leqq 0$. Since in this case $\lambda$ and $\lambda$ are decreasing functions of $\kappa(\S 2)$, and $\bar{\lambda}$ and $\bar{\lambda}$ are increasing functions, there can be at most two intersections, one for positive values of $\lambda$ and one for negative values.

In cases (1) and (2) it is evident that not every line within one of the sets of half-lines lies in the other. For the existence of solutions of $(A)$ of the type sought it is then necessary that there exist two values $\bar{\kappa}$ and $\overline{\bar{\kappa}}$ such that $A_{11}+\bar{\kappa} A_{12}$ has one sign and $A_{21}+\bar{\kappa} A_{22}$ is zero or of contrary sign for at least one value of $y$ and that $A_{21}+\overline{\bar{k}} A_{22}$ has one sign and $A_{11}+\overline{\bar{k}} A_{12}$ is zero or of contrary sign for at least one value of $x$. The statement of the sufficient conditions is the same except that zero is excluded. That the solution in case (1) be unique and in case (2) double, it is sufficient that there exist a value $\kappa^{\prime}$ such that $A_{11}+\kappa^{\prime} A_{12}, A_{21}+\kappa^{\prime} A_{22}$ have each one sign and those opposite.

By a linear transformation of the parameters $\lambda, \mu$ the directions $\bar{\kappa}, \overline{\bar{\kappa}}$ may be chosen as the axes. The normal form of the equations $(A)$ in case there are solutions of the type sought is then

$$
\begin{array}{ll}
A_{11} \geqq 0 \text { for every } x, & A_{12}<0 \text { for some } x ; \\
\mathrm{A}_{21}<0 \text { for some } y, & A_{22} \geqq 0 \text { for every } y .
\end{array}
$$

Brown University, Providence, R. I. 